Short Communication

\title{
CHOANAL ATRESIA- A CLINICAL STUDY AND REVIEW OF LITERATURE
}

\author{
Satheesh Kumar Bhandary ${ }^{1}$, Vadisha Bhat ${ }^{2} \&$ Shwetha Shenoy $M .^{3}$ \\ ${ }^{1}$ Professor \& Head, ${ }^{2}$ Associate Professor, ${ }^{3}$ Senior Resident, Department of E.N.T, K.S. Hegde M edical Academy, \\ Nitte University, Mangalore - 575 118, India \\ Correspondence : \\ Satheesh Kumar Bhandary \\ Vice Dean, K. S. Hegde M edical Academy, M angalore- 575018, Karnataka
}

\begin{abstract}
:
Choanal atresia is a developmental anomaly where there is failure of communication of the nasal cavity to the nasopharynx. The atresia can be membranous or bony in nature, but may be mixed as is seen in most of the cases. Bilateral choanal atresia is an emergency condition and newborns can have severe respiratory distress and cyanosis at birth. Failure to pass an $8 \mathrm{~F}$ catherter through the nasal cavity more than $5.5 \mathrm{~cm}$ from the alar rim points to the presence of choanal atresia. In emergency, bilateral choanal atresia is managed with an oropharyngeal airway. Flexible nasal endoscopy and CT scan can confirm the diagnosis of choanal atresia. Surgery is the definitive treatment which can be done either by transnasal or transpalatal approach. The transnasal endoscopic route is currently the preferred procedure and can be performed as a minimally invasive procedure. This has less complications and a high success rate. We studied four cases of unilateral choanal atresia presented to our hospital, with respect to the etiological factors, clinical features and management.
\end{abstract}

Keywords: Choanal atresia; Unilateral; Endoscopic recanalization

\section{Introduction:}

Choanal atresia is caused by persistence of the buccopharyngeal membrane during development. It was first described by Roederer in 1755. The reported incidence is between 1 in 5000 to 9000 live births. ${ }^{1}$ In many cases choanal atresia associated with other anomalies. The most common among these is CHA R GE association

( $\mathrm{C}=$ coloboma, $\mathrm{H}$ =heart disease, $\mathrm{A}=$ atresia of choanae, $\mathrm{R}=$ retarded growth and development, $\mathrm{G}=$ genital hypoplasia, $E$ = ear deformities or deafness). Other anomalies associated with choanal atresia include polydactyly, nasalauricular and palatal deformities, Crouzon's syndrome, Down syndrome, Treacher-Collins syndrome, DiGeorges yndrome, craniosynostosis, microencephaly, meningocele, meningoencephalocele, facial asymmetry, hypoplasia of the orbit and midface, cleft palate, and hypertelorism. ${ }^{2}$

Choanal atresia is conventionally classified in two types, bony $(90 \%)$ and membranous (10\%). A more recent analysis suggests a mixed bony/membranous in $70 \%$ and pure bonyin $30 \%$.

Choanal atresia may be unilateral or bilateral. Bilateral
Atresia is an emergency condition requiring instant restoration of airway. Whereas unilateral choanal atresia may be managed electively as these patients will not have respiratory distress. M ost of the cases of unilateral choanal atresia are diagnosed while evaluating the patient for causes of unilateral nasal obstruction and discharge

We have studied four cases of Choanal atresia presented to the department of ENT of K S Hegde Hospital, Deralakatte, Mangalore, Karnataka between January 2008 and December 2011.

\section{Materials and Methodology:}

Patients of Choanal Atresia presented to our hospital with features of persistent unilateral nasal obstruction and discharge or patients who were evaluated and diagnosed elsewhere and referred to our institution for management were taken into the study. Patients presented with complains of unilateral nasal obstruction were evaluated clinically, and with endoscopy and CT scan to confirm the presence of Choanal atresia. They were also evaluated by Pediatricians, Ophthalmologists for the presence of syndromic associations and ECHO cardiogram was done wherever necessary. Three of these patients were 
managed by endoscopic recanalization using zero degree endoscope. Posterior septal mucosa was incised after infiltration with 1: 200000 adrenaline in $2 \%$ lignocaine solution. Septal mucoperichondrium was elevated posteriorly, from medial to lateral. Posterior part of the bony septum was removed and the two nasal cavities were joined posteriorly. The atretic plate was widened by removing the bone using Kerrisons punch and soft tissues were removed using microdebrider and nasal cavity is joined to the nasopharynx. No stent was placed. Nasal cavity was packed with merocel sponge and was removed after 48 hours. Endoscopic cleaning was done after one week. Patients were followed up by Diagnostic nasal endoscopy weekly for one month and later once in a month for sixmonths, and later once in every six months.

In one patient with Aperts syndrome, surgical management was deferred as the patient was two years of age and he did not have any respiratory distress.

\section{Observations:}

Four cases of Choanal atresia in last three years between 2008 and 2011 were studied. Three were males and one was female. Youngest was a two year old male. Oldest in our study was a 20 year old female, who had undergone surgery for choanal atresia at the age of 12 years and was referred to us for revision surgery, as she had developed restenosis.

Case \#1: A 10 year old boy presented with the history of right nasal obstruction since birth and persistent nasal discharge from the right nasal cavity. On general examination, deviation of the angle of the mouth to the left on smiling was noted. Eye closure and forehead wrinkling was normal. Child was also found to have grade II malnutrition. His nasal framework was normal. Absent misting on the right was noted on cold spatula test. Anterior rhinoscopy showed mucoid nasal discharge on the right side. Posterior rhinoscopy revealed right choanal obstruction. His right ear was low- set with microtia. Both tympanic membranes were normal but tuning fork test revealed bilateral mixed hearing loss. His right Tonsil was smaller compared to the left. He was evaluated for the presence of CHARGE association. Aortic stenosis was noted on Echocardiogram. Audiometry confirmed mixed hearing loss on both the sides. This child was diagnosed to have right Hemifacial microsomia with CHARGE association (Fig 1). Computed Tomography scan showed features of bony atresia of the right choana (Fig 2). This child underwent Endoscopic recanalization of choanal atresia. Postoperative period was uneventful and the choana is patent after 2 years of follow up.

Case \#2: A 13 year old boy presented with history of right nasal discharge since childhood, which is present continuously. He was diagnosed to have right sided choanal atresia at three months of age and was advised surgery after 10 years by hisfamily physician. On general evaluation there were no syndromic features. Anterior rhinoscopy showed mucoid discharge in the right nasal cavity. Posterior rhinoscopy showed atresia of the right choana. Submucous cleft palate and bifid uvula was noted on examination of the oral cavity. Cardiac evaluation was normal. Patient underwent endoscopic recanalization and is presently asymptomatic and is under regular follow up for 1 year.

Case \#3: A 20yr old, who was diagnosed with choanal atresia was referred to us for revision surgery for choanal atresia. She had undergone surgery for choanal atresia at the age of 12 years by transnasal approach, but developed restenosis (Fig 3). Oral cavity examination showed a midline sub mucosal cleft palate, coloboma of both the eyelids, flattened nasal bridge and hypertelorism. She had characteristic CHARGE ear pinna anomaly on the right side. We did endoscopic recanalization for her. She is asymptomatic at present one year after surgery.

Case \#4: A 2yr old boy presented with history of right sided nasal obstruction and nasal discharge. On examination boy had flat occiput, flat frontal bone, steeple shaped cranial vault, hypertelorism, bilateral syndactyly, macroglossia, microstomia, and bilateral axial proptosis. We could not pass an $8 \mathrm{~F}$ catheter through the right nasal cavity into the nasopharynx. CT scan showed right sided bony choanal atresia. The child was diagnosed to have Aperts syndrome 
with Choanal atresia (Fig 4). As the patient was not having any respiratory distress, the surgical management for choanal atresia was deferred.

\section{Discussion:}

Choanal atresia is the closure of the posterior choanae of the nose. The persistence of the buccopharyngeal membrane from the foregut is one of the most acceptable theories of atresia of the choana and choanal stenosis.

Other proposed theories include failure of perforation of the nasobuccal membrane of Hochstetter; abnormal persistence or location of mesoderm forming adhesions in the nasochoanal region; or misdirection of neural crest cell migration.
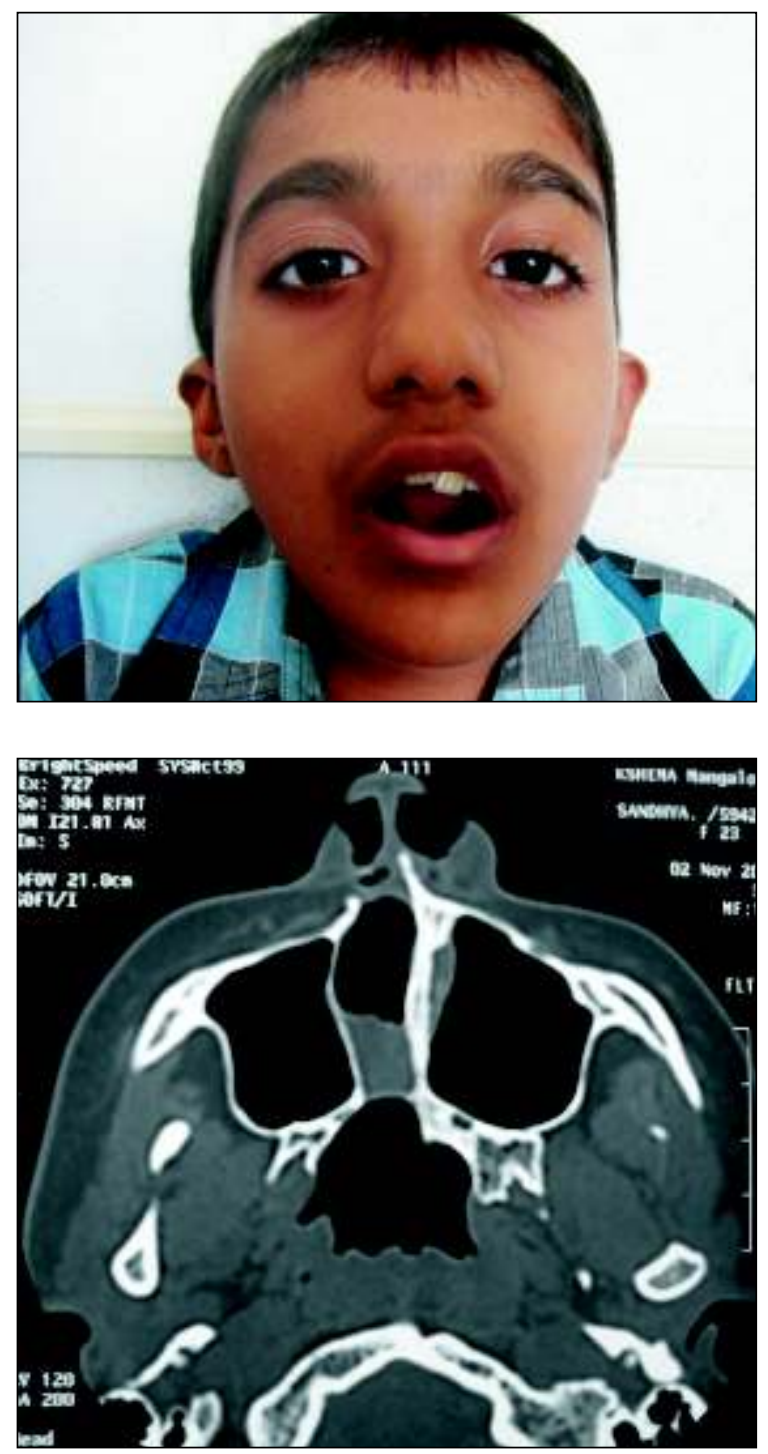

Recent theories state that abnormalities in vitamin $\mathrm{A}$ metabolism are associated with choanal atresia. Prenatal use of thionamides (e.g. methimazole or carbimazole) has been associated with choanal atresia in the offspring. ${ }^{1}$

Thirty percent of the children with choanal atresia had CHARGE syndrome. The CHARGE association was first described in 1979 by Hall and Hittner who evaluated a series of patients with choanal atresia and multiple anomalies. Pagon et al first coined the term CHARGE association (Coloboma, Heart defect, Atresia choanae, Retarded growth and development, Genital hypoplasia, Ear anomalies/deafness). Presently, the major which include the classical four Cs: Choanal atresia, Coloboma,
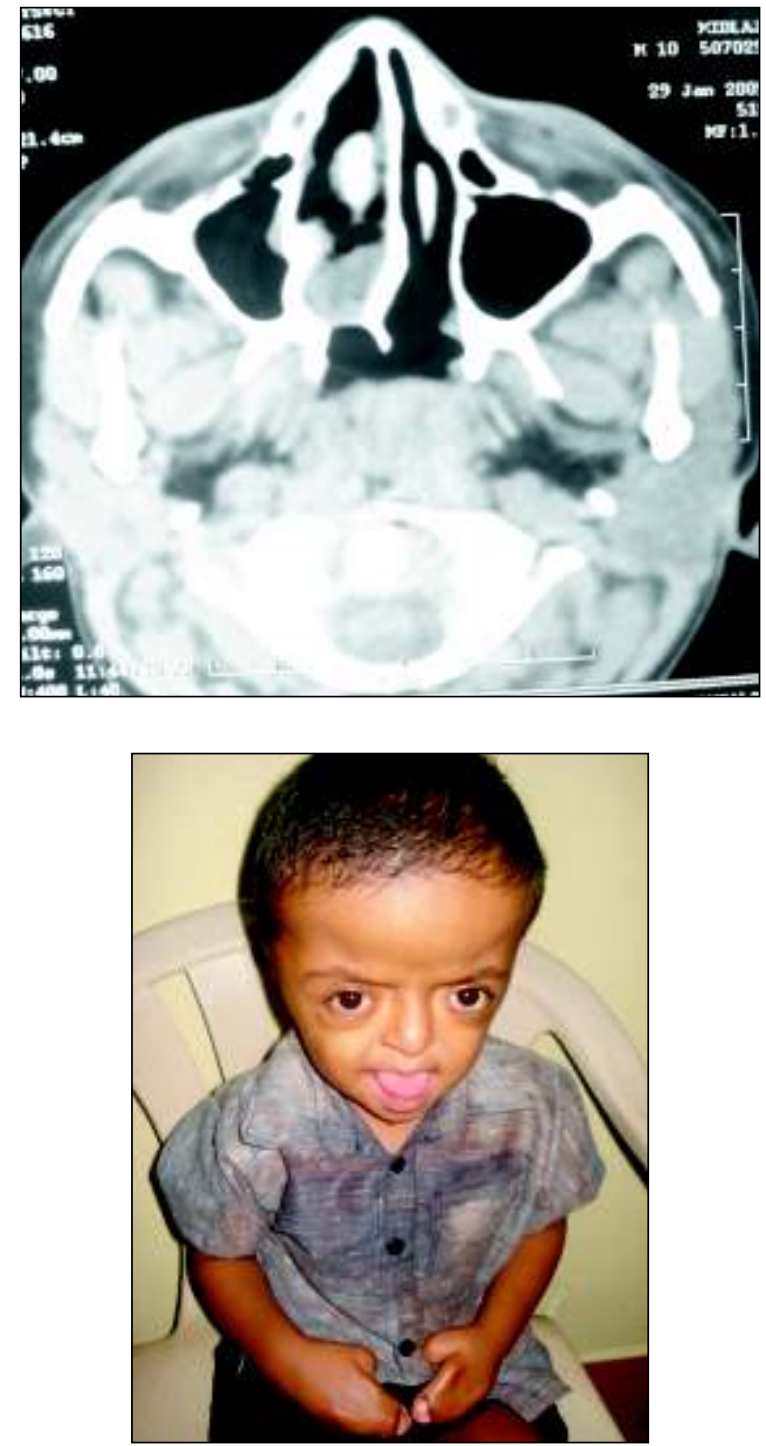
Characteristic ears and Cranial nerve anomalies including Sensory neural hearing loss and the minor features including Cardio-vascular malformations, Genital hypoplasia Pubertal delay, Short stature, Cleft lip, Tracheoesophageal fistula, Reflux esophagitis; and developmental delay have been defined for the clinical diagnosis of CHARGE syndrome. ${ }^{3}$

In all four of our patients unilateral choanal atresia was present on the right side. Two of our patients had CHARGE association, one had Aperts syndrome and one did not have any syndromic association.

M anagement of Choanal atresia: Bilateral choanal atresia presents respiratory depression in a newborn. This is seen because neonates are obligate nasal breathers. They present as cyclical cyanosis which relieves on crying. Placement of appropriate sized oral airway can temporarily manage the respiratory emergency. Endotracheal intubation may be required.

Management of unilateral choanal atresia is not an emergency as respiration is not compromised. Transnasal and transpalatal surgery are the two options available.

Transnasal repair can be done under direct vision and a 120 degree endoscope placed behind the soft palate to visualize the nasopharynx. The atretic plate is perforated and widened with a microdebrider. Stents made of Portex are placed with non absorbable sutures to maintain patency ${ }^{4}$. This method had high chance of restenosis and surgical failure.

Endoscopic transnasal surgery was first attempted by Stankiewicz. The endoscope is used to perforate the atretic plate either under direct vision through the nose, or more commonly, using dilators observed via the postnasal space with mirror or 120 degree endoscope. After a passage is made into the postnasal space, the opening is enlarged, typically by removing the posterior part of the septum. Injury to the turbinates must be avoided as they result in synechiae. ${ }^{5}$

Restenosis is prevented by the use of Mitomycin C, an antiproliferative agent that inhibits fibroblast growth and proliferation. It is prevents scar tissue and granulation formation. However it has fallen to dispute because of its potential oncogenic capacity. ${ }^{6}$

Use of stents to maintain patency has been attempted. Stenting is advisable in children who have a higher risk of failure like in neonates and bilateral choanal stenosis.

Sinha et al described a technique of puncture of choanal atresia using Hegars dilator and stenting with portex endotracheal tube and achieved good results. ${ }^{7}$

Injury to the alar and septal cartilages or palate may result in necrosis of the cartilages is the problem associated with stenting. The maximum size dilator should not exceed the size of the anterior nares. The maintenance of patency of the stents is of concern because of recurrent obstruction due to crusting and need for frequent monitored irrigation. ${ }^{8}$

In our technique, with the aid of endoscope, the posterior part of the bony nasal septum was removed and the two nasal cavities are combined and the atretic plate is removed joining the nasal cavity to the nasopharynx. We had not placed stents. The complication of restenosis was not seen in any of the patients in our study.

Transpalatal surgery allows correction of all of the anatomical abnormalities, including the medialization of the lateral wall at the anterior pterygoid plates. This procedure had a high rate of palatal abnormalities in the growing period. ${ }^{1}$

\section{Conclusion:}

Choanal atresia presents with respiratory distress when bilateral and persistent nasal discharge when unilateral. An otorhinolaryngologist has to be familiar with the situation and rule of choanal atresia. Choanal atresia is also commonly associated with other congenital anomalies which have to be evaluated and managed accordingly. The presence of associated congenital anomalies poses challenge in the management of choanal atresia. Endoscopic transnasal approach is a minimally invasive technique and has less chance of complications and restenosis. 
References:

1. James D. Ramsden, Paolo Campisi , Vito Forte. Choanal Atresia and Choanal Stenosis. Otolaryngol Clin N Am (2009)42; 339-352.

2. Assanasen P, Metheetrairut C. Choanal Atresia. J Med Assoc Thai(2009) Vol. 92 No. 5

3. Kim D Blake, Chitra Prasad. CHARGE syndrome. Orphanet Journal of Rare Diseases (200); 1:34.

4. Gujarati CS, Daniel SJ, James AL, et al. Management of bilateral choanal atresia in the neonate: an institutional review. Int J Pediatr Otorhinolaryngol (2004); 68 (4):399-407.

5. Stankiewicz JA. Pediatric endoscopic nasal and sinus surgery.
Otolaryngol Head Neck Surg (1995)113(3):204-10.

6. Agrawal $\mathrm{N}$, Morrison $\mathrm{GA}$. Laryngeal cancer after topical mitomycin $\mathrm{C}$ application.J Laryngol Otol(2006);120(12):1075-6.

7. Sinha V, Sinha S, Gupta D, More Y, Prajapati B, Bhat V et al. Choanal Atresia : Surgery by Puncture, Dilation and Stenting. J Rhinol 2006; 13(2): 124-7

8. Al-Ammar AY. The use of nasal stent for choanal atresia. Saudi Med J 2008; 29(3): 437-40. 